\author{
Devachandran Jayakumar \\ R. Pratheema \\ Nagarajan Ramakrishnan
}

\section{Ulcerative necrosis of the uvula following endotracheal intubation}

Received: 19 February 2015

Accepted: 21 February 2015

Published online: 7 March 2015

(C) Springer-Verlag Berlin Heidelberg and ESICM 2015

D. Jayakumar $(\bowtie) \cdot$ R. Pratheema $\cdot$ N. Ramakrishnan Apollo Speciality Hospital - OMR, Chennai, Tamil Nadu, India e-mail: drdevachandran_j@apollohospitals.com

A 22-year-old man was admitted to ICU with features suggestive of fat embolism syndrome, 3 days after tibial nailing under general anaesthesia. The intubation for anaesthesia was uneventful and he was extubated at the end of the procedure, which lasted $2 \mathrm{~h}$. In the ICU, he complained of severe, persistent sore throat with foreign body sensation in the throat. A throat examination revealed ulcerative necrosis of the uvula with a clear demarcation between the necrotic area and the remaining uvula (Fig. 1).

Ulcerative necrosis of the uvula is an uncommon complication of endotracheal intubation. Pressure over the uvula from an endotracheal tube placed in the midline or the uvula getting folded over itself can compromise the blood supply causing ischaemic ulceration and necrosis. If the uvula gets caught in the suction cannula during extubation, it can also cause ischaemic oedema, which leads to necrosis. Since our patient had a clear demarcation between the necrotic area and the rest of the uvula, it was probably caught in the suction cannula during suctioning. With conservative management, his sore throat

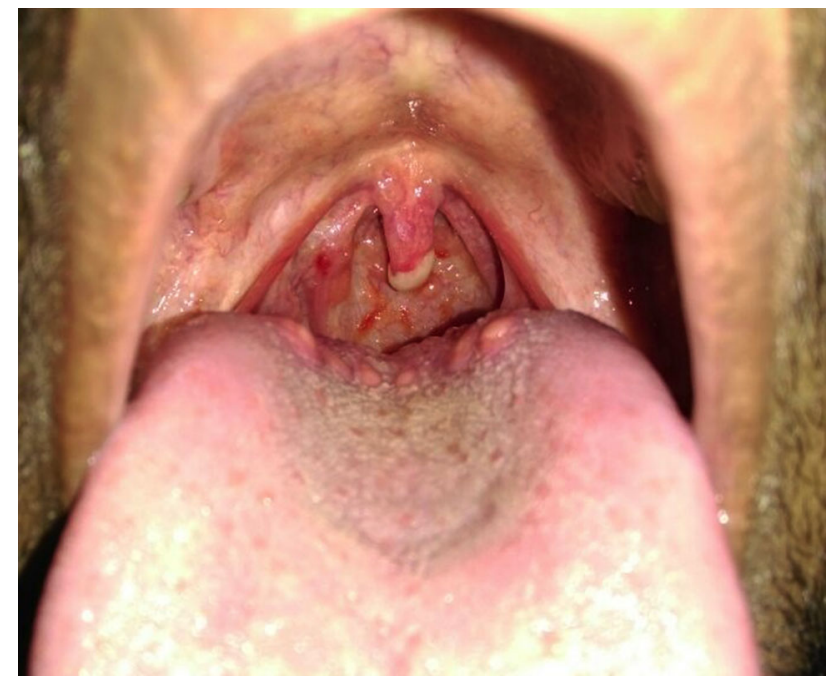

Fig. 1 Picture showing the ulcerative necrosis

resolved in 3 days and his uvula had healed completely when he came for follow-up 13 days later.

Apart from endotracheal intubation, there are also reports of uvular necrosis after endoscopy and transoesophageal echocardiography. Positioning the endotracheal tube or the endoscope away from the uvula and taking extra care while suctioning will prevent this complication.

Conflicts of interest None.

Informed consent Obtained. 